\title{
Dosimetric Evaluation of Total Body Irradiation (TBI) Treatment by Volumet- ric Modulated Arc Therapy (VMAT) on the Coach
}

\author{
Tas B*, Durmus IF, Okumus A, and Uzel OE
}

Department of Radiation Oncology, Yeni Yuzyil University Gaziosmanpasa Hospital, Istanbul, Turkey

${ }^{*}$ Corresponding author: Tas B, Department of Radiation Oncology, Yeni Yuzyil University Gaziosmanpasa Hospital, Istanbul, Turkey, E-mail: bora_tash@yahoo.com

Citation: Tas B, Durmus IF, Okumus A, Uzel OE (2017) Dosimetric Evaluation of Total Body Irradiation (TBI) Treatment by Volumetric Modulated Arc Therapy (VMAT) on the Coach. J Biophy Biochem 1(1): 103

Received Date: November 28, 2016 Accepted Date: March 24, 2017 Published Date: March 27, 2017

\begin{abstract}
To compare three different types of volumetric modulated arc therapy (VMAT) planning tecniques using Elekta Versa HD lineer accelerator to deliver total body irradition (TBI) treatment on the coach and to evaluate feasibility of VMAT planning tecnique for TBI treatment.

Five TBI patient's treatment planning performed using Monaco5.1 ${ }^{\circ}$ treatment planning system with three different VMAT tecniques for each patient. First one was single arc VMAT tecnique, second one was dual arc VMAT tecnique and third one was 2 fields arc VMAT tecnique for one isocentre. The VMAT-TBI tecnique consisted of three isocentres and three overlapping arcs. Eight TBI patient's treatment planning system (TPS) was performed with the best VMAT technique which we determined. The prescribed dose was $12 \mathrm{~Gy}$. Mean dose to lungs and kidneys were restricted less than $10 \mathrm{~Gy}$ and maximum dose to lens were restricted less than $6 \mathrm{~Gy}$. X-ray volume imaging (XVI) cone beam computer tomography (CT) was used as an image guided radiation therapy (IGRT) method for each VMAT delivery, in addition, C-RAD surface guided radiotherapy (SGRT) device was used for patient positioning and monitoring. The plans were verified using 2 Dimensional array and ion chamber for patient quality assurance (QA). The comparison between calculation and measurement were made by $\gamma$-index analysis and absolute dose.

This study demonstrates that dual arc VMAT technique has got less monitor units (MUs) than other techniques but an average delivery time is \%17 more than single arc VMAT technique. When we compared organ at risk (OAR)s, we had less dose and better dose coverage to target with dual arc VMAT technique. An average total delivery time was determined $923 \pm 34$ seconds and an average MU was determined $2614 \pm 228$ MUs for eight TBI patient's dual arc VMAT. Mean dose to lungs was $9.7 \pm 0.2$ Gy, mean dose to kidneys was $8.8 \pm 0.3 \mathrm{~Gy}$, maximum dose to lens was $5.5 \pm 0.3 \mathrm{~Gy}$ and maximum point dose to patient was $14.6 \pm 0.3 \mathrm{~Gy}$, hetereogenity index (HI) of planning target volume (PTV) was $1.13 \pm 0.2$, mean dose to PTV was $12.6 \pm 1.5$ Gy and mean $\gamma$-index pass rate was $\% 97.1 \pm 1.9$.

The results show that the tecnique for TBI using VMAT on the treatment coach is feasible with dual arc VMAT tecnique on the coach. Keywords: TBI, VMAT
\end{abstract}

\section{Introduction}

Total body irradiation (TBI) is designed to irradiate the whole body uniformly to a prescribed dose while minimizing radiation induced complications to lungs and other normal organs. TBI with megavoltage photon beams is one component used in treating several diseases, including multiple myeloma, leukemias, lymphomas and some solid tumors $[1,2]$. Also, conjuction with chemotherapy is widely used as a conditioning regimen before bone marrow transplant (BMT) for patients with hematologic malinancies. In combination with chemotherapy demonstrates superior treatment outcomes for BMT conditioning compared with chemotherapy alone [3-7]. TBI provides a uniform dose of radiation to the entire body, penetrating areas such as the central nervous system (CNS) and testes, where traditional chemotherapy is ineffective [8]. The purpose of this treatment is threefold: to eliminate residual cancer cells, to provide space for stem cell engraftment through bone marrow depletion, and to prevent rejection of donor stem cells through immunosuppression $[9,10]$.

Studies in mice and humans show that the toxicities of TBI can be improved further by fractionating the radiation. The rate of lung repair between fractions was reviewed by Travis [11], indicating the presence of two significantly different repair rates corresponding to a fast repair half time of 0.40 hours and a slow half time of 4.01 hours. The slow repair component needs to be kept in mind when designing TBI schedules that include two or three fractions per a day [12]. The ideal dosing schedule depends on patient age, disease and the intended type of stem cell transplant [7]. Recommended dose schedule for myeloablative TBI is 12 
to 15 Gy given in 8 to 12 fractions over 4 days, with 2 to 3 treatments daily doses $>15$ Gy have been shown to decrease relapse rate, but also increase the incidence of graft vs. host disease and decrease 2 years survival [8]. Low-dose TBI, with doses of 2 to 8 Gy given in 1 to 4 fractions in combination with chemotherapy, is an effective conditioning regimen for hematopoietic stem cell transplantation in patients who cannot tolerate myeloablation due to age or comorbidities $[8,13,14]$. A randomized study from Seattle in the setting of AML compared single dose TBI (10 Gy) to a fractionated schedule (2 Gy for six fractions). The last update of this trial showed significant superiority of the fractioanated scheme in terms of every free survival [15]. Another Seattle randomized trial of AML in first remission compared fractionated TBI doses of 12 Gy with 15.75 Gy, showing a decreased relapse rate from $35 \%$ to $12 \%$, but at the expense of a significant increase in therapy related mortalit, resulting in no survival advantages to a higher radiation dose [16].

Many different tecniques have been described for effective irradiation of the whole body and indeed improvements in both the irradation tecnique and physical dosimetry. Most of the current TBI procedures are based on tecniques established on linear accelerators that are used for conventional radiotherapy. The large photon fields are generally achieved by treating the patient at extended SSD with standart linear accelerators or with special dedicated machines. The variable SSD available at current radiotherapy facilities is partially responsible for the variation in TBI tecniques and procedures. Equipment Guidelines recommend the use of parallel opposed pairs of high-energy photon beams from 4 to $18 \mathrm{MV}$ for TBI [2]. TBI tecniques typically comprise a combination of various opposing field setups in a sitting or lying patient position at very extended SSDs. The extended SSD tecnique using a single large field encompassing the entire patient is by far the simplest and the most prevalent TBI tecnique used today. It eliminates the dosimetry complications in regions of field junctions introduced by using multiple small fields and concern that cells circulating through the body could potentially receive a reduced dose. These tecniques use Standard radiotherapy linear accelerators (LINAC)s and rely on a maximum collimator setting, a large SSD, and beam divergence to produce the large irradiation field required for TBI. AAPM's TG-51 calibration protocol provides guidelines for dosimetry of high-energy photon beams [17]. Recent studies demonstrate the efficacy of helical tomotherapy for decreasing TBI treatment time and increasing homogeneity of delivered radiation [18]. Helical tomotherapy (HT) allows for irradiation of large target volume at nominal source axis distance (SAD), without additional requirements on treatment room size or shielding. HT consists of a linac mounted on a computed tomography (CT) gantry and delivers a fan beam (up to $5 \mathrm{~cm} \mathrm{x} 40 \mathrm{~cm}$ ) modulated with binary 64-leaf multileaf collimator (MLC). The beam source continuously rotates in the gantry while the couch is translated through the bore, which generates a helical pattern that can cover a treatment length of up to $160 \mathrm{~cm}$. Because of their limited body length the tecnique seems especially eligible in juvenile patients.

In our study, we investigated the use of Linac-Based TBI by VMAT at nominal SAD technique on the coach. Five TBI patient's treatment planning performed using Monaco5. $1^{\circledR}$ treatment planning system with three different VMAT tecniques for each patient. First one was single arc VMAT tecnique, second one was dual arc VMAT tecnique and third one was 2 fields arc VMAT tecnique for one isocentre. The VMAT-TBI tecnique consisted of three isocentres and three overlapping arcs. Eight TBI patient's TPS were performed with the best VMAT technique which we determined.

\section{Materials and Methods}

For treating patients, Versa $\mathrm{HD}^{\circledR}$ (Crawley, Elekta) linear accelerator with $6 \mathrm{MV}$, equipped with Agility ${ }^{\circledR}$ collimator system, XVI 5.0 cone beam CT was used as an image guided radiation therapy (IGRT) method for each VMAT delivery, also, Sentinel ${ }^{\circledR}$ (Sweeden, C-RAD) laser based surface guided radiotherapy (SGRT) device was used for patient positioning and monitoring. This is shown Figure 1.

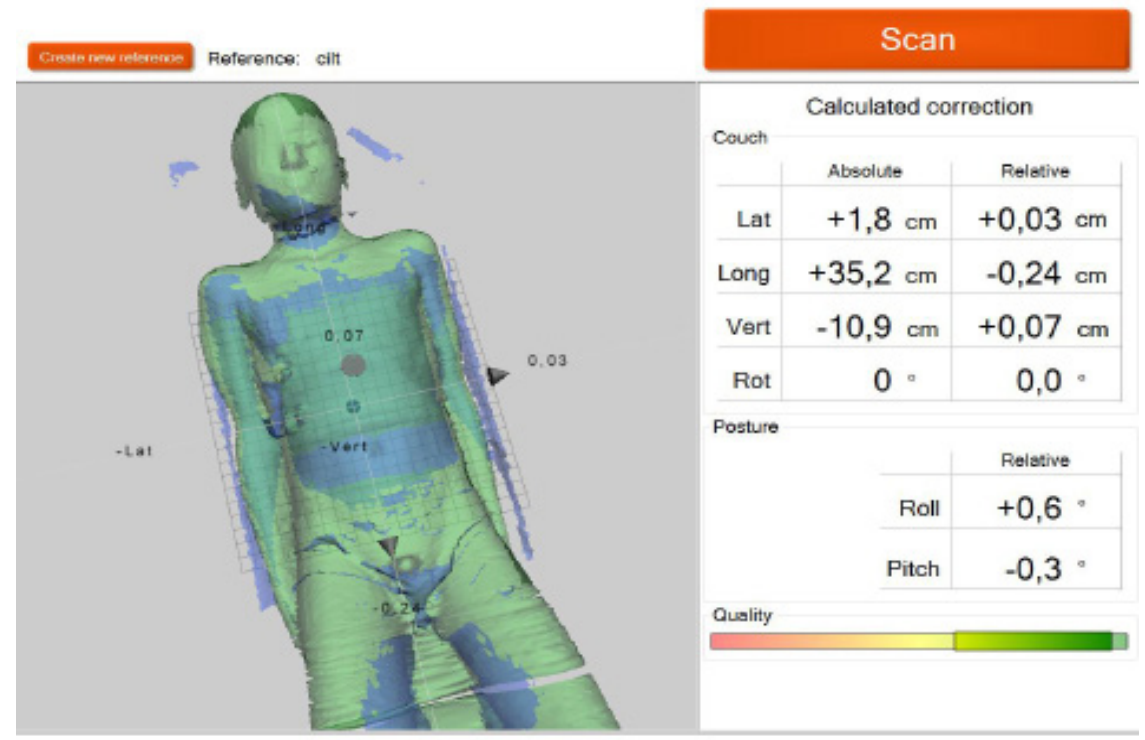

Figure 1: One TBI patient's positioning and monitoring by Sentinel laser based SGRT scan 
Agility ${ }^{\oplus}$ collimator system included 160 MLC, minimum leaf width was $5 \mathrm{~mm}$, maximum field size was $40 \mathrm{x} 40 \mathrm{~cm}^{2}$ at nominal SAD:100 cm. MLC's effective speed was $6.5 \mathrm{~cm} /$ second and leaf travel was $15 \mathrm{~cm}$ over the central axis. VMAT plans were generated on Monaco $5.1^{\oplus}$ (Crawley, Elekta) treatment planning system with Monte Carlo algorithm. All calculation parameters were grid spacing $0.3 \mathrm{~cm}$, minimum segment width $1.0 \mathrm{~cm}$, maximum 180 of control points per arc, fluence smoothing medium, statistical uncertainty $1.0 \%$ per calculation, increment of gantry $30^{\circ}$ and calculate dose deposition to medium.

All patients were positioned supin with head toward the gantry. The VMAT-TBI tecnique consisted of three isocentres and three dual overlapping arcs. $75 \%$ of patients were pediatric, $\% 25$ of patients were adult and all patients were male in our study. We have treated adult patients with three dual overlapping arcs to the bottom of pelvic bone then we were positioned with feet toward the treatment gantry and we used 2 more overlapping arcs for whole body treatment. The prescribed dose was $90 \%$ of target volume receiving dose of $12 \mathrm{~Gy}$. Mean dose to lungs and kidneys were restricted less than 10Gy and maximum dose to lens were restricted less than 6 Gy. The plans were verified using 2 Dimensional array IBA Matrixx ${ }^{\oplus}$ patient quality assurance (QA) system and CC13 ion chamber. The comparison between calculation and measurement were made by $\gamma$-index ( $3 \%-3 \mathrm{~mm})$ analysis and absolute dose measurement at the isocentre.

\section{Results}

When we compared five patient's organ at risk (OAR)s doses for three VMAT techniques, we had less dose and better dose coverage to target with dual arc VMAT technique, it is shown Table 1. Segments, total MUs and duration of treatment comparison between VMAT tecniques are given Table 2. Also, Figure 2 shows dose distribution of one TBI treatment by dual VMAT technique on the coach.

\begin{tabular}{|c|c|c|c|c|c|c|c|c|}
\hline & \multicolumn{4}{|c|}{ Single arc VMAT } & \multicolumn{3}{c|}{ Two fields arc VMAT } \\
\hline Patient & Lung R. & Lung L. & Kidney R. & Kidney L. & Lung R. & Lung L. & Kidney L. & Kidney L. \\
\hline 1 & $9.6 \%$ & $6.0 \%$ & $8.6 \%$ & $9.7 \%$ & $11.0 \%$ & $8.6 \%$ & $12.0 \%$ & $9.6 \%$ \\
\hline 2 & $11.0 \%$ & $12.0 \%$ & $7.0 \%$ & $13.0 \%$ & $16.0 \%$ & $10.0 \%$ & $10.0 \%$ & $17.0 \%$ \\
\hline 3 & $1.8 \%$ & $1.6 \%$ & $3.5 \%$ & $-1.0 \%$ & $4.7 \%$ & $4.5 \%$ & $4.0 \%$ & $4.0 \%$ \\
\hline 4 & $4.2 \%$ & $4.0 \%$ & $6.7 \%$ & $7.6 \%$ & $-2.2 \%$ & $0.0 \%$ & $5.0 \%$ & $4.8 \%$ \\
\hline 5 & $3.8 \%$ & $5.0 \%$ & $8.4 \%$ & $5.0 \%$ & $2.5 \%$ & $2.9 \%$ & $0.1 \%$ & $0.2 \%$ \\
\hline Mean \pm SD & $6.08 \pm 4.0 \%$ & $5.72 \pm 3.9 \%$ & $6.84 \pm 2.0 \%$ & $6.86 \pm 5.3 \%$ & $6.40 \pm 7.1 \%$ & $5.20 \pm 4.1 \%$ & $6.22 \pm 4.8 \%$ & $7.12 \pm 6.4 \%$ \\
\hline
\end{tabular}

Table 1: Mean doses of lungs and kidneys difference between dual arc VMAT and other tecniques

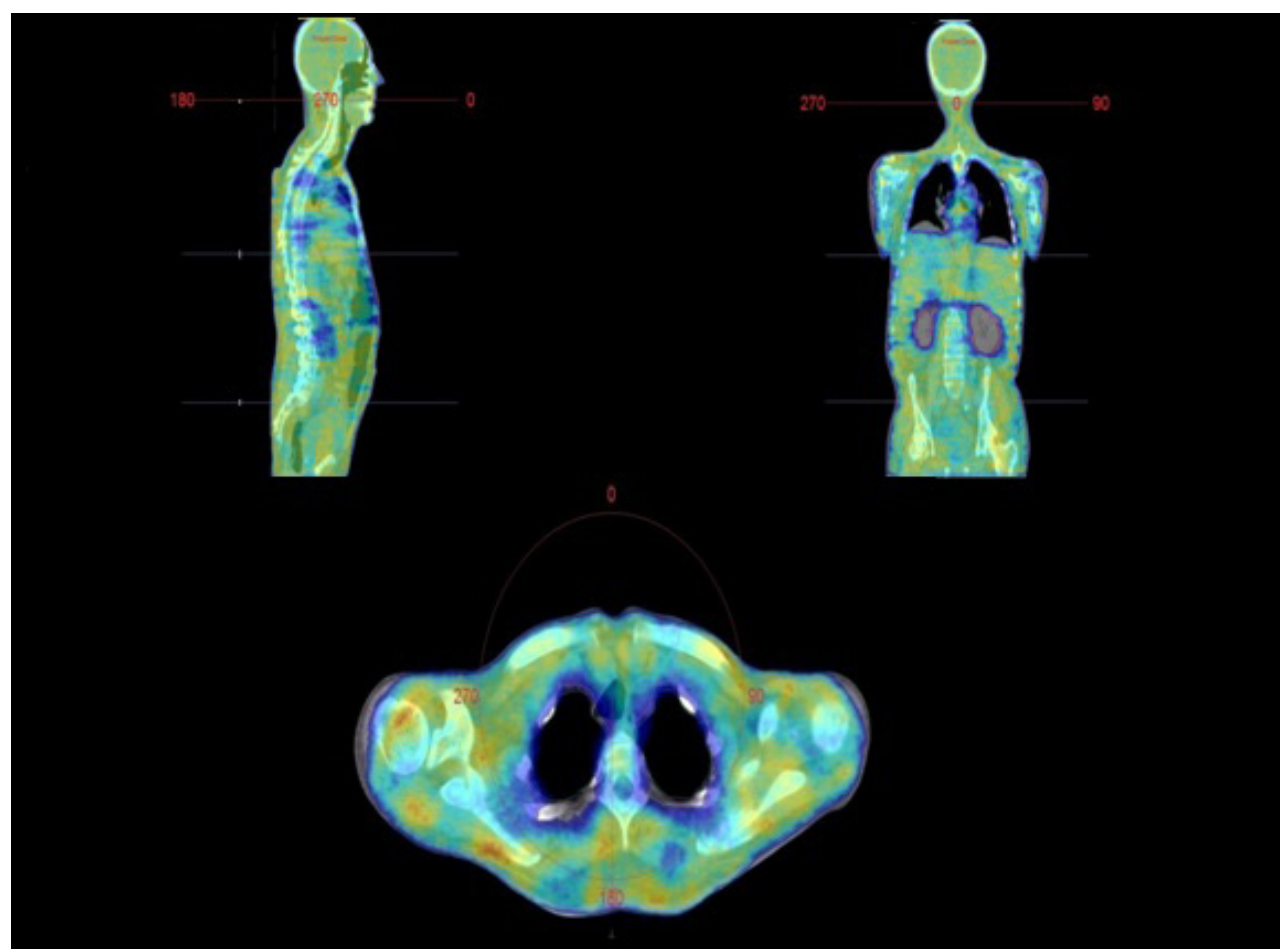

Figure 2: Dose distribution of TBI treatment's sagital, coronal and axial plane by dual arc VMAT tecnique 


\begin{tabular}{|c|c|c|c|c|c|c|c|c|c|}
\hline & \multicolumn{3}{|c|}{ Dual arc VMAT } & \multicolumn{3}{c|}{ Single arc VMAT } & \multicolumn{3}{c|}{ Two fields arc VMAT } \\
\hline Patient & Segments & Total MUs & $\begin{array}{c}\text { Duration } \\
\text { (seconds) }\end{array}$ & Segments & Total MUs & $\begin{array}{c}\text { Duration } \\
\text { (seconds) }\end{array}$ & Segments & Total MUs & $\begin{array}{c}\text { Duration } \\
(\text { seconds.) }\end{array}$ \\
\hline 1 & 651 & 1903.33 & 847.56 & 452 & 2202.01 & 695.27 & 802 & 2992.15 & 1305.90 \\
\hline 2 & 641 & 2286.86 & 723.55 & 422 & 2788.57 & 661.05 & 785 & 3299.35 & 1233.83 \\
\hline 3 & 670 & 2042.11 & 800.69 & 473 & 2900.35 & 732.04 & 879 & 3653.44 & 1432.15 \\
\hline 4 & 753 & 2573.09 & 908.33 & 456 & 2962.72 & 733.65 & 851 & 3298.61 & 1514.01 \\
\hline 5 & 723 & 2207.01 & 895.84 & 473 & 2562.15 & 732.57 & 839 & 3397.92 & 1476.58 \\
\hline Mean \pm SD & $687.60 \pm 48$ & $2202.48 \pm 255$ & $835.19 \pm 76$ & $455.20 \pm 21$ & $2683.16 \pm 309$ & $710.90 \pm 32$ & $831.20 \pm 38$ & $3328.29 \pm 237$ & $1392.49 \pm 118$ \\
\hline
\end{tabular}

Table 2: Number of segments, total MU's and duration of treatment's comparison between VMAT tecniques

An average total delivery time was determined $923 \pm 34$ seconds and an average monitor unit (MU)s was determined $2614 \pm 231$ MU for dual arc VMAT technique. When we evaluated organ at risk (OAR)s, mean dose to lungs was $9.73 \pm 0.2 \mathrm{~Gy}$, mean dose to kidneys was $8.89 \pm 0.3 \mathrm{~Gy}$, maximum dose to lens was $5.50 \pm 0.3 \mathrm{~Gy}$ and maximum point dose was $14.59 \pm 0.3 \mathrm{~Gy}$, HI of PTV was $1.13 \pm 0.02$, mean dose to PTV was $12.60 \pm 0.15 \mathrm{~Gy}$ and mean $\gamma$-index $(3 \%-3 \mathrm{~mm})$ pass rate was $\% 97.13 \pm 1.9$. These are shown Table 3.

\begin{tabular}{|c|c|c|c|c|c|c|c|c|c|c|c|c|}
\hline Patient & $\begin{array}{l}\text { Lung R. } \\
\text { (Gy) }\end{array}$ & $\begin{array}{l}\text { Lung L. } \\
\text { (Gy) }\end{array}$ & $\begin{array}{l}\text { Kidney } \\
\text { R. (Gy) }\end{array}$ & $\begin{array}{l}\text { Kidney } \\
\text { L. (Gy) }\end{array}$ & $\begin{array}{l}\text { Lens R. } \\
\text { (Gy) }\end{array}$ & $\begin{array}{l}\text { Lens L. } \\
\text { (Gy) }\end{array}$ & $\begin{array}{l}\text { Max. Dose } \\
\text { (Gy) }\end{array}$ & $\begin{array}{l}\text { Mean Dose } \\
\text { of PTV (Gy) }\end{array}$ & HI & $\gamma$-index & Total MUs & $\begin{array}{l}\text { Beam On } \\
\text { (seconds) }\end{array}$ \\
\hline 1 & 9.9 & 9.4 & 8.7 & 8.6 & 5.3 & 5.1 & 14.3 & 12.6 & 1.11 & $\% 96.2$ & 2665.8 & 953.9 \\
\hline 2 & 9.4 & 9.6 & 8.6 & 8.8 & 5.3 & 5.3 & 14.3 & 12.5 & 1.12 & $\% 94.3$ & 2248.3 & 901.4 \\
\hline 3 & 10.0 & 9.9 & 8.9 & 9.0 & 6.0 & 5.7 & 14.9 & 12.5 & 1.16 & $\% 99.2$ & 2564.2 & 874.3 \\
\hline 4 & 9.9 & 9.8 & 9.0 & 9.3 & 5.8 & 6.0 & 14.8 & 12.7 & 1.14 & $\% 97.6$ & 2838.6 & 950.4 \\
\hline 5 & 9.8 & 9.7 & 9.3 & 8.7 & 5.8 & 5.6 & 14.6 & 12.6 & 1.14 & $\% 98.2$ & 2756.8 & 937.1 \\
\hline 6 & 9.7 & 9.8 & 8.8 & 9.0 & 5.2 & 5.2 & 14.5 & 12.4 & 1.14 & $\% 98.1$ & 2804.6 & 921.6 \\
\hline 7 & 9.5 & 9.6 & 8.9 & 8.6 & 5.4 & 5.3 & 14.4 & 12.6 & 1.12 & $\% 94.7$ & 2706.6 & 883.4 \\
\hline 8 & 9.8 & 9.7 & 8.8 & 9.2 & 5.3 & 5.6 & 14.9 & 12.9 & 1.13 & $\% 98.7$ & 2604.6 & 961.9 \\
\hline Mean \pm SD & $9.75 \pm 0.2$ & $9.69 \pm 0.2$ & $8.88 \pm 0.2$ & $8.90 \pm 0.3$ & $5.51 \pm 0.3$ & $5.48 \pm 0.3$ & $14.59 \pm 0.3$ & $12.60 \pm 0.15$ & $1.13 \pm 0.02$ & $\% 97.13 \pm 1.9$ & $2648.69 \pm 231$ & $923 \pm 34$ \\
\hline
\end{tabular}

Table 3: Dosimetric features of TBI by dual arc VMAT tecnique

\section{Conclusion}

The results show that dose coverage of target and OAR's doses also depend significantly VMAT techniques. MLCs mostly move just one side of isocentre in first tour of VMAT then MLCs mostly move other side of isocentre in second tour of VMAT with dual arc VMAT technique, therefore this technique is decreasing MUs and delivery time of treatment, also a benefit could be demonstrated with regard to dose distribution and homogeneity and dose-reduction to organs at risk. We could use cone beam CT images and SGRT method for positioning and monitoring patients. The tecnique for TBI using dual arc VMAT on the treatment coach was found feasible. Additionally, we determined highly precise dose delivery by TBI patient's quality assurace (QA) and point dose measurement. Total Marrow Irradiation (TMI) treatment is also possible with VMAT based treatment on the coach by linear accelerator. Based on the dose distributions we have decided to plan TBI in our clinic with dual arc VMAT technique on the treatment coach.

\section{References}

1. Zheng Y, Dou Y, Duan L, et al. (2015) Using chemo-drugs or irradiation to break immune tolerance and facilitate immunotherapy in solid cancer. Cellular Immunology 294: 54-9.

2. Levitt, SH, Purdy JA, Perez CA (2012) Technical Basis of Radiation Therapy: Practical Clinical Applications. Berlin, Germany: Springer 31: $786-97$.

3. Ringdén O, Labopin M, Tura S, Arcese W, Iriondo A, et al. (1996) A comparison of Busulphan versus total body irradiation combined with cyclophosphamide as conditioning for autgraft or allograft bone marrow transplantation in patients with acute leukemia. Acute Leukemia Working Party of the European Group for Blood and Marrow Transplantation (EBMT). Br J Haematol 93: 637-45.

4. Davies SM, Ramsay NK, Klein JP, Weisdorf DJ, Bolwell B, et al.(2001) Comparison of preparative regimes in transplants for children with acute lymhoblastic leukemia. J Clin Oncol 18: 340-7.

5. Kröger N, Zabelina T, Krüger W, Renges H, Stute N, et al. (2001) Comparison of total body irradiation vs busulfan in conbination with cyclophosphamide as conditioning for enrelated stem cell transplantation in CML patients. Bone Marrow Transplant 27: 349-54.

6. Sampath S, Schultheiss TS, Wong J (2005) Dose response and factors related to interstitial pneumonitis after bone marrow transplant. Int J Radiat Oncol Biol Phys 63: 876-84.

7. Shank B, Hopfan S, Kim JH, Chu FC, Grossbard E, et al. (1982) Hyperfractionated total body irradiation for bone marrow transplantation: I. Early results in leukemia patients. Int J Radiat Oncol Biol Phys 7: 1109-15.

8. Seung S, Larson D, Galvin J, Mehta MP, Potters L, et al. (2013) American College of Radiology (ACR) and American Society for Radiation Oncology (ASTRO) Practice Guideline for the Performance of Stereotactic Radiosurgery (SRS). Am J Clin Oncol 36: 310-15. 
9. Halperin E, Constine L, Tarbell N, Kun L. (2005) Pediatric Radiation Oncology 4th Edition. Philadelphia: Lippincott Williams \& Wilkins.

10. Halperin E, Wazer D, Perez C, Brader L (2013) Perez and Brady's Principles and Practice of Radiation Oncology 6th Edition. Philadelphia: Lippincott Williams \& Wilkins.

11. Travis EL (1980) The sequence of histological changes in Mouse lungs after single doses of x-rays. Int J Radiat Oncol Biol Phys 6: $345-7$.

12. Vegesna V, Withers HR, Thames HD Jr, Mason K (1985) Multifraction radiation response of Mouse lung. Int J Radiat Biol Relat Stud Phys Chem Med 47: 41322.

13. Storb R, Raff RF, Appelbaum FR, Deeg HJ, Graham TC, et al. (1994) Fractionated versus single-dose total body irradiation at low and high dose rates to condition canine littermates for DLA-identical marrow grafts. Blood 83: 3384-9.

14. Storb R, Raff RF, Appelbaum FR, Graham TC, Schuening FG, et al. (1989) Comparison of fractionated to single-dose total body irradiation in conditioning canine littermates for DLA-identical marrow grafts. Blood 74: 1139-43.

15. Thomas ED (1990) Total body irradiation regimes for marrow grafting. Int J Radiat Oncol Biol Phys 19: 1285-8.

16. Clift RA, Buckner CD, Appelbaum FR, Bearman SI, Petersen FB, et al. (1990) Allogeneic marrow transplantation in patients with acute myeloid leukemia in first remission: a randomized trial of two irradiation regimens. Blood 76: 1867-71.

17. McEwen M, Dewerd L, Ibbott G, et al. (2014) Addendum to the AAPM's TG-51 Protocol for Clinical Reference Dosimetry of High-energy Photon Beams. Med Phys 41: 041501.

18. Hui SK, Kapatoes J, Fowler J, Henderson D, Olivera G, et al. (2005) Feasibility study of helical tomotherapy for total body or total marrow irradiation. Med Phys 32: 3214- 24.

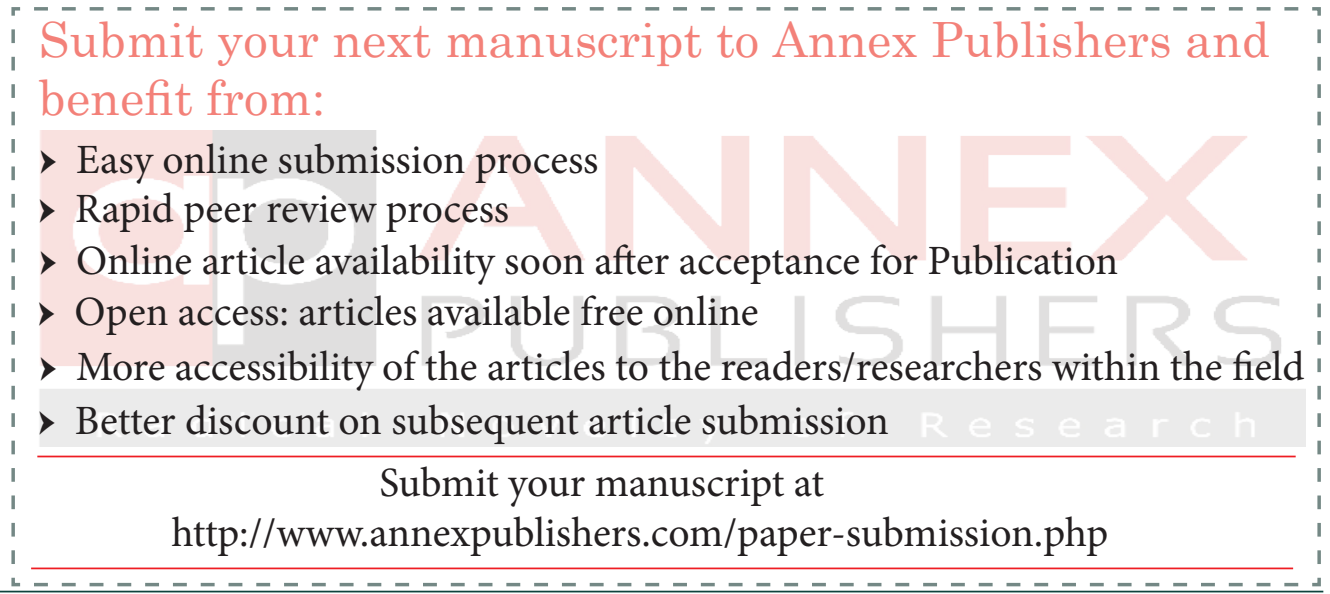

